\title{
छs \\ Orbital tomography: Deconvoluting photoemission spectra of organic molecules
}

\author{
P. Puschnig,,${ }^{1,}$ E.-M. Reinisch, ${ }^{2}$ T. Ules, ${ }^{2}$ G. Koller, ${ }^{2}$ S. Soubatch,${ }^{3,4}$ M. Ostler, ${ }^{5}$ L. Romaner, ${ }^{1}$ F. S. Tautz, ${ }^{3,4}$ \\ C. Ambrosch-Draxl, ${ }^{1}$ and M. G. Ramsey ${ }^{2}$ \\ ${ }^{1}$ Chair of Atomistic Modelling and Design of Materials, Montanuniversität Leoben, 8700 Leoben, Austria \\ ${ }^{2}$ Institute of Physics, Karl-Franzens Universität Graz, 8010 Graz, Austria \\ ${ }^{3}$ Peter Grünberg Institut (PGI-3), Forschungszentrum Jülich, 52425 Jülich, Germany \\ ${ }^{4}$ Jülich Aachen Research Alliance, Fundamentals of Future Information Technology, 52425 Jülich, Germany \\ ${ }^{5}$ Lehrstuhl für Technische Physik, Universität Erlangen-Nürnberg, 91058 Erlangen, Germany \\ (Received 16 September 2011; revised manuscript received 14 November 2011; published 15 December 2011)
}

\begin{abstract}
We study the interface of an organic monolayer with a metallic surface, i.e., PTCDA (3,4,9,10-perylenetetracarboxylic-dianhydride) on $\mathrm{Ag}(110)$, by means of angle-resolved photoemission spectroscopy (ARPES) and $a b$ initio electronic structure calculations. We present a tomographic method that uses the energy and momentum dependence of ARPES data to deconvolute spectra into individual orbital contributions beyond the limits of energy resolution. This provides an orbital-by-orbital characterization of large adsorbate systems without the need to invoke a sophisticated theory of photoemission, allowing us to directly estimate the effects of bonding on individual orbitals. Moreover, these experimental data serve as a most stringent test necessary for the further development of $a b$ initio electronic structure theory.
\end{abstract}

DOI: 10.1103/PhysRevB.84.235427

PACS number(s): 73.20.-r, 79.60.-i, 71.15.Mb

\section{INTRODUCTION}

The energy of molecular orbitals and their energy ordering are of fundamental importance in research fields ranging from surface chemistry to organic opto-electronics. The valence bands of large conjugated molecules consist of a multiplicity of closely spaced molecular states, and their correct assignment is challenging both experimentally and theoretically. Experimentally, energy positions of molecular orbitals in organic molecular layers can be studied by ultraviolet photoemission spectroscopy (UPS ${ }^{1}$ or by scanning tunneling spectroscopy (STS). ${ }^{2-7}$ UPS has the advantage that accessible binding energies are not limited to a few electron volts from the Fermi level as in STS. However, UPS spectra of thin molecular layers on metals often show only weak and rather broad features and are, therefore, not always conclusive. Also, UPS data depend on the experimental geometry, molecular orientation, and photon energy, which further complicates the assignment of the measured peaks. Therefore, calculated densities of states (DOSs) from ab initio electronic-structure calculations are commonly used to complement and interpret UPS data. However, this is problematic, for various reasons: First, in density-functional theory (DFT) calculations, both the orbital energies and the orbital-energy order rely on approximations made for exchange-correlation effects, and thus the functional chosen.$^{8-11}$ Second, due to computational limitations, more elaborate theoretical methods that go beyond DFT, such as wave-function-based methods or many-body perturbation theory, are difficult to apply to organic monolayers adsorbed on metallic substrates. Third, strictly speaking, individual experimental energy distribution curves (EDCs) cannot be interpreted as DOSs, since the measurement process inevitably involves transition probabilities that emphasize or suppress

\footnotetext{
Published by the American Physical Society under the terms of the Creative Commons Attribution 3.0 License. Further distribution of this work must maintain attribution to the author(s) and the published article's title, journal citation, and DOI.
}

certain states depending on experimental conditions and molecular arrangement.

In this work we present a tomographic analysis of angleresolved photoemission spectroscopy (ARPES) that provides a solution to the problem of assigning spectral features in UPS to specific molecular states. We collect ARPES data in the full half-space above the sample surface. This data set contains a complete momentum- and energy-resolved DOS of the sample in the measured binding-energy range. To assign emissions to specific molecular states, we evaluate tomographic cross sections through the momentum-resolved data set and search for momentum space patterns that are characteristic for the corresponding molecular orbitals. This analysis is based on a simple plane-wave approximation for the final state in the photoemission process that has been shown to be appropriate for many $\pi$-conjugated organic molecules ${ }^{12-15}$ since scattering effects have been demonstrated to be negligible. ${ }^{16}$ In this approximation, the ARPES intensity distribution from a molecular state is related to the respective molecular orbital's Fourier transform. The combination of these simulated momentum maps with experimental tomographs allows the quantitative deconvolution of an EDC into contributions from individual molecular states beyond the limits of intrinsic line broadening and instrumental energy resolution, thus even if its deconvolution based on conventional peak fitting is impossible. This is achieved by carrying out the deconvolution in $k$ space, thereby making use of the full momentum space information of the molecular orbitals. The so-obtained orbital projected DOS provides a stringent test for electronic-structure theories including density functional calculations as well as wave-function-based approaches.

\section{METHODS}

\section{A. Experimental setup}

Photoemission experiments are performed at BESSYII with $30 \mathrm{eV}$ photons using a toroidal electron-energy analyzer that simultaneously records photoelectrons emitted with polar 
angles of $-80^{\circ}$ to $+80^{\circ}$ with respect to the surface normal. For our tomographic analysis of angle-resolved photoemission spectroscopy, we collect ARPES data in the full half-space above the sample surface by stepwise rotating the sample azimuthally over $180^{\circ}$. This acquisition of the photoemission data cube requires the use of display-type detectors. For our ARPES experiments a toroidal electron energy analyzer as described previously ${ }^{17}$ was attached to the beamline U125/2-SGM of the synchrotron radiation facility BESSY II, Helmholtz-Zentrum-Berlin. A photon energy of $30 \mathrm{eV}$ and a photon incidence angle of $40^{\circ}$ with respect to the surface normal were used. The polarization direction is in the specular plane, which also contains the photoelectron trajectory measured. The toroidal energy analyzer collects the photoelectrons emitted in a kinetic energy window of $0.8 \mathrm{eV}$ width over a $\pm 80^{\circ}$ polar angle $(\theta)$ range simultaneously. Note, however, that we take only the data from the side of the emission direction that is pointing toward the electric field vector of the incident photons, i.e., $\theta=0^{\circ}$ to $+80^{\circ}$. Thereby, the polarization factor appearing in the photocurrent cross section [see Eq. (3)] is maximized, leading to a better signal-to-noise ratio. To obtain the constant binding-energy (CBE) slabs over the full $\left(k_{x}, k_{y}\right)$ range, azimuthal scans are made by rotating the sample around the surface normal in $1^{\circ}$ steps for $>180^{\circ}$ of azimuthal angle $\phi$ and then imposing the substrate's twofold symmetry to obtain the full $360^{\circ}$. The angular emission data are then converted to parallel momentum components $k_{x}$ and $k_{y}$ :

$$
\begin{aligned}
& k_{x}=\sqrt{2 m_{e} E_{\mathrm{kin}} / \hbar^{2}} \sin \theta \cos \phi, \\
& k_{y}=\sqrt{2 m_{e} E_{\mathrm{kin}} / \hbar^{2}} \sin \theta \sin \phi
\end{aligned}
$$

to create the momentum maps (CBE slabs). An individual CBE slab has an energy width of $0.8 \mathrm{eV}$ and consists of 40 individual slices, which goes well below the analyzer's intrinsic energy resolution of $150 \mathrm{meV}$. The full photoemission data cube is now assembled by collecting a sequential series of CBE slabs. Note that a $0.2 \mathrm{eV}$ overlap at the slab boundary was used for normalization purposes.

The $\operatorname{Ag}(110)$ substrate was prepared in the conventional way by a sequence of sputter-annealing cycles. PTCDA molecules were evaporated from an effusion cell at $510 \mathrm{~K}$ onto the $\mathrm{Ag}(110)$ surface at room temperature. The structure of PTCDA/Ag(110) was controlled by LEED to avoid the appearance of compressed monolayer reflexes and thus to preserve the single domain order of the film with molecules oriented in only one direction.

\section{B. Computational details}

Electronic structure calculations are performed within density functional theory (DFT) for both the free PTCDA molecule as well as the adsorbed monolayer by using a variety of exchange-correlations functionals. For the free PTCDA molecule, we utilize ABINIT. ${ }^{18}$ The all-electron potentials are replaced by extended norm-conserving, highly transferable Troullier-Martins pseudopotentials ${ }^{19}$ using a plane-wave cutoff of 50 Ryd. We employ a super cell approach with a box size of $50 \times 50 \times 25 \mathrm{Bohr}^{3}$ and $\Gamma$ point sampling of the Brillouin zone. The geometry of the free molecule is optimized by using a generalized gradient approximation $(\mathrm{GGA})^{20}$ for exchange-correlation effects.

Orbital energies are calculated by using various approximations for exchange-correlation effects: (1) A GGA, ${ }^{20}$ (2) a self-interaction corrected GGA (SIC), ${ }^{9}(3)$ the screened hybrid functional according to Heyd and co-workers (HSE), ${ }^{21,22}$ and (4) $G W$ calculations at the $G_{0} W_{0}$ level. Self-interaction corrections (SICs) to GGA orbital energies are treated according to Eq. (2) of Ref. 9, which is based purely on quantities obtained within the local density approximation (LDA). Self-consistent calculations of the free PTCDA molecule using a screened hybrid functional HSE06 ${ }^{21,22}$ are performed with the VASP code. ${ }^{23,24}$ The calculation of $G W$ corrections are obtained at the $G_{0} W_{0}$ level by utilizing the ABINIT package. ${ }^{18}$ Here we use a cubic supercell of size $32 \times 32 \times 32$ Bohr $^{3}$ and cut off the Coulomb potential outside a sphere of radius 16 Bohr. $^{25}$ We utilize a plasmon pole model ${ }^{26}$ and the energy effective technique to reduce the sum over empty states. ${ }^{27}$ The cutoff for wave functions and dielectric matrices are chosen to be 50 and 6 Ryd, respectively, which resulted in $G_{0} W_{0}$ corrections to be converged within $0.1 \mathrm{eV}$.

All calculations for the PE intensity are done for free PTCDA molecules assuming a plane-wave final state. ${ }^{28}$ As outlined in more detail in a previous paper, ${ }^{14}$ and noted earlier, ${ }^{29,30}$ these approximations lead to the simple result that the PE intensity $\phi_{i}$ from a given initial state $i$ is proportional to the square modulus of the Fourier transform of the initial-state wave function $\tilde{\psi}(\mathbf{k})$ :

$$
\phi_{i}\left(k_{x}, k_{y} ;|\mathbf{k}|\right) \propto(\mathbf{A} \cdot \mathbf{k})^{2}\left|\tilde{\psi}_{i}\left(k_{x}, k_{y} ;|\mathbf{k}|\right)\right|^{2} .
$$

Here $\mathbf{k}$ and $\mathbf{A}$ are the wave vector of the emitted electron and the vector potential of the incident photon, respectively. Note that in this work we do not attempt to recover the phase information of the Fourier transform that would be necessary if one were interested in reconstructing wave functions from photoemission data. ${ }^{30,31}$ The dependence $\left(k_{x}, k_{y} ;|\mathbf{k}|\right)$ in Eq. (3) means that a hemispherical cut through the three-dimensional Fourier transform has to be carried out with a radius $|\mathbf{k}|$ determined by the kinetic energy of the final-state electron. This simple approach in combination with an appropriate experimental geometry has been shown to describe experimental data for a number of systems in an excellent manner, ${ }^{12-15}$ even though it neglects effects such as multiple scattering, which has, however, been shown to have only negligible effects even in the presence of transition metal atoms. ${ }^{16}$ It has the added advantage of enabling a one-to-one relation between the angular PE patterns and the Fourier transform of the initial state orbital. We also stress that while orbital energies are quite sensitive to the treatment of exchange-correlation effects, the corresponding wave functions are much less so, ${ }^{11}$ which we have tested by comparing self-consistent orbitals obtained from GGA and HSE06 calculations. Hence, the simulated PE momentum maps, which are proportional to the Fourier transforms of these orbitals, are quite insensitive to the choice of exchange-correlations functional. Thus, our tomographic approach to obtain projected density-of-states curves from ARPES momentum maps is quite robust with respect the treatment of exchange-correlation effects. 
Electronic structure calculations for the $c(4 \times 6)$ PTCDA/Ag(110) interface have been carried using the VASP $\operatorname{code}^{23,24}$ using a generalized gradient approximation for exchange-correlation effects. ${ }^{20}$ The $\mathrm{Ag}(110)$ substrate is modeled by five layers of $\mathrm{Ag}$ with an additional vacuum layer of $16 \AA$. The projector augmented waves (PAW) ${ }^{32}$ approach was used allowing for a relatively low-kinetic-energy cutoff of about $285 \mathrm{eV}$. We use a Monkhorst-Pack $8 \times 8 \times 1$ grid of $k$ points, ${ }^{33}$ and a first-order Methfessel-Paxton smearing of $0.2 \mathrm{eV}^{34}$ To avoid spurious electrical fields, a dipole layer is inserted in the vacuum region. ${ }^{35}$ In order to circumvent issues concerning van-der-Waals interactions, which are ill-described in standard GGA functionals, ${ }^{36,37}$ we take the heights of the molecular carbon backbone as well as the heights of the carboxylic and anhydride oxygen atoms above the $\mathrm{Ag}$ substrate from $\mathrm{x}$-ray standing-wave experiments. ${ }^{38}$ In the geometry optimization, we allow only for relaxations parallel to the surface. Since in standard DFT codes molecular orbital information is lost from the calculated DOS, we also evaluate the projection of the DOS onto the molecular orbitals. ${ }^{39}$ These have been carried out as described previously ${ }^{37,39,40}$ using the SIESTA code. ${ }^{41}$ The most detailed explanation on how to calculate the DOS projected onto molecular orbitals is provided in a previous publication. ${ }^{40}$

\section{RESULTS}

\section{A. Energy distribution curve}

We demonstrate our tomographic method by applying it to PTCDA (3,4,9,10-perylene-tetracarboxylic-dianhydride) on $\mathrm{Ag}$, arguably one of the most studied large-molecule adsorbate systems exhibiting both model character and relevance for device applications. ${ }^{42}$ Specifically, the $c(4 \times 6)$ monolayer on a $\operatorname{Ag}(110)$ surface is well suited since all molecules are aligned parallel to the [001] azimuth. ${ }^{43,44}$ Figure 1(a) displays an ARPES band map with the take-off plane at $32^{\circ}$ with respect to the substrate's [001] direction and binding energies $\left(E_{b}\right)$ ranging from the Fermi level to $-8 \mathrm{eV}$. When looking at the corresponding $k$-integrated energy distribution curve (EDC), i.e., the white line in Fig. 1(a), we observe three features that appear upon adsorption of the molecule, denoted as M1, M2, and M3. They are found at binding energies of $-0.8,-1.9$, and $-3.4 \mathrm{eV}$, respectively. Below these relatively weak peaks, emissions from the $\mathrm{Ag} 4 d$ bands dominate. Following the commonly accepted practice of assigning UPS peaks to specific molecular states, we compare the energy positions of M1, M2, and M3 to orbital energies obtained from $a b$ initio electronic-structure calculations. This is done in Fig. 1(b), using various calculations for the free molecule. Note that we align all computed HOMO levels with M2 at $-1.9 \mathrm{eV}$ and color-code $\pi$ and $\sigma$ orbitals in black (dark) and orange (light gray), respectively. As mentioned above, both the orbital energies and their ordering as obtained from DFT are prone to functional-dependent errors. Nevertheless, it seems reasonable to tentatively assign M2 to the HOMO and M1 to the filled LUMO, since it is known from STS experiments that upon adsorption on $\operatorname{Ag}(110)$ the LUMO of PTCDA is filled by charge transfer from the metal. ${ }^{3}$ The situation for M3, however, remains unclear, because a large

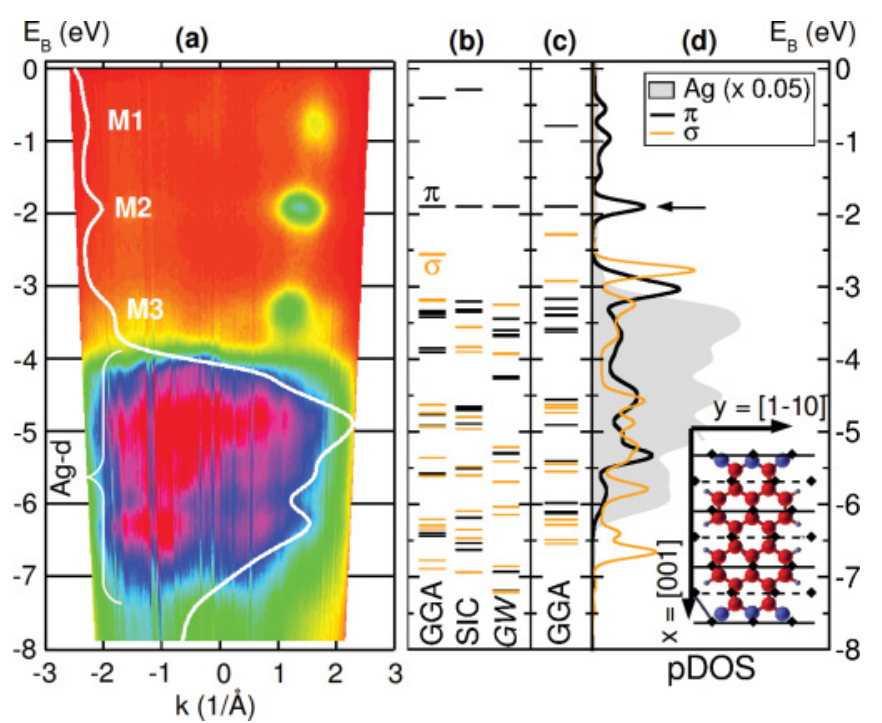

FIG. 1. (Color online) (a) Experimental band map of PTCDA/Ag (110) for an emission plane rotated by $32^{\circ}$ with respect to the $\mathrm{Ag}$ [001] direction. The white line is the $k$-integrated energy distribution curve (EDC). Molecular features (M1, M2, and M3) as well as the $\mathrm{Ag} d$ bands are indicated. (b) Calculated orbital energies of a free PTCDA molecule from a generalized gradient approximation (GGA), a self-interaction corrected GGA (SIC), and a $G W$ calculation. (c) GGA orbital energies of a free PTCDA molecule in the geometry of the adsorbed monolayer on $\mathrm{Ag}(110)$. (d) Projected density of states (PDOS) of PTCDA/Ag(110) (experimental geometry) using GGA. Projections on Ag atoms (shaded gray, multiplied by 0.05 ), and molecular $\pi$ (black) and $\sigma$ (orange) states are shown. The inset displays the adsorption geometry; the long molecular axis of PTCDA $(x)$ is parallel to the [001] substrate direction.

number of states is predicted in this binding-energy range. Moreover, the generalized gradient approximation (GGA) predicts the HOMO- 1 to be a $\sigma$ orbital concentrated on the anhydride groups and located in the gap between M2 and M3, which disagrees with gas-phase photoemission spectra. ${ }^{8}$ By correcting the GGA for its well-known self-interaction error, either by using hybrid functionals or self-interaction-corrected functionals (SIC) $)^{9,10}$ or by performing $G W$ calculations, ${ }^{8}$ this $\sigma$ orbital is shifted to higher binding energies, thereby apparently improving the agreement with experiment.

However, to be precise one should compare the experimental EDC in Fig. 1(a) to calculations that take into account the geometrical distortion of PTCDA upon adsorption ${ }^{45}$ and the hybridization with substrate states, since both may lead to shifts and broadenings of molecular states. In Figs. 1(c) and 1(d) we show GGA orbital energies of a free PTCDA molecule in the distorted geometry that it acquires on $\operatorname{Ag}(110)^{38}$ and a GGA-DOS plot of a PTCDA monolayer on $\mathrm{Ag}(110)$ in the experimental geometry. ${ }^{38} \mathrm{We}$ observe shifts of orbital energies; in particular, the anhydride $\sigma$ states move to even lower binding energies. For PTCDA adsorbed on $\mathrm{Ag}(110)$, the DOS projected onto molecular orbitals ${ }^{39}$ reveals a broadened state close to the Fermi level, presumably arising from the filled LUMO, and below a sharper peak, which could arise from the HOMO and which we therefore align with M2. But as for the free molecule, the GGA results for PTCDA/Ag(110) yield the anhydride $\sigma$ (orange line) in the gap between M2 and M3, and the first 


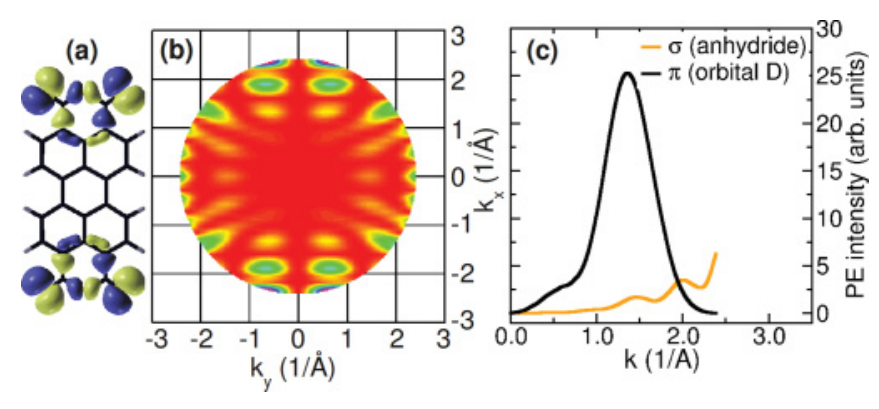

FIG. 2. (Color online) (a) Molecular $\sigma$ orbital localized at the oxygen atoms of PTCDA and (b) its momentum map using a finalstate kinetic energy of $22.4 \mathrm{eV}$. (c) Comparison of the simulated, azimuthally averaged photo current of this $\sigma$ state (orange) with a typical $\pi$ orbital at a similar binding energy (dark line).

$\pi$ states below the HOMO appear too high in energy. An additional problem is connected to the fact that GGA predicts the $\mathrm{Ag} 4 d$ states about $1 \mathrm{eV}$ too high compared to experiment.

\section{B. Classification of PTCDA orbitals}

Following the above discussion, we must conclude that a reliable assignment of the peaks M1, M2, and, in particular, M3, based on binding-energy arguments alone, is impossible. Therefore, we make use of the pronounced $k$ dependence of molecular emissions as found in the energy-distribution map in Fig. 1(a). M1, M2, and M3 seem to belong to one molecular " $\pi$-band," similar to what has been observed for sexiphenyl. ${ }^{12}$ This finding already allows us to exclude the anhydride $\sigma$ orbital to be the origin for M3. This is also evident from Fig. 2, where we display a real space picture of the anhydride $\sigma$ molecular orbital localized at the oxygen atoms together with its momentum map according to Eq. (3) using a final-state kinetic energy of $22.4 \mathrm{eV}$. In order to make a quantitative comparison between the photoemission intensity from this $\sigma$ state and a typical $\pi$ state, we also compare the azimuthally averaged photo current of the anhydride $\sigma$ state (orange line) with a typical $\pi$ orbital at a similar binding energy (black). One can see that the PE intensity arising from the anhydride $\sigma$ orbital is roughly a factor of 10 smaller than that of the $\pi$ orbital, a general finding for flat-lying, $\pi$-conjugated molecules. Moreover, states that are more localized in real space, such as the anhydride $\sigma$, appear more diffuse in reciprocal space, i.e., have a larger spread in momentum space as compared to delocalized $\pi$ states with a pronounced nodal structure. Overall, the anhydride $\sigma$ orbital does not exhibit a significant photoemission intensity around the $k$ value of $1.1 \AA^{-1}$, where M3 is peaked, as shown in Fig. 1(a). This leads us to conclude that M1, M2, and M3 must all arise from $\pi$ states.

The molecular $\pi$ orbitals of PTCDA can be classified according to their symmetry and momentum distribution. ${ }^{14}$ The result is summarized in Fig. 3, where, based on their nodal structure, we group $12 \pi$ orbitals into three bands. The first band, $\pi_{1}$, is characterized by a $x z$ nodal plane through the long molecular axis $(x)$ and consists of five orbitals with an increasing number of nodal planes perpendicular to $x$. This is reflected in the corresponding momentum maps, in which the main peaks disperse to higher $k_{x}$ values with

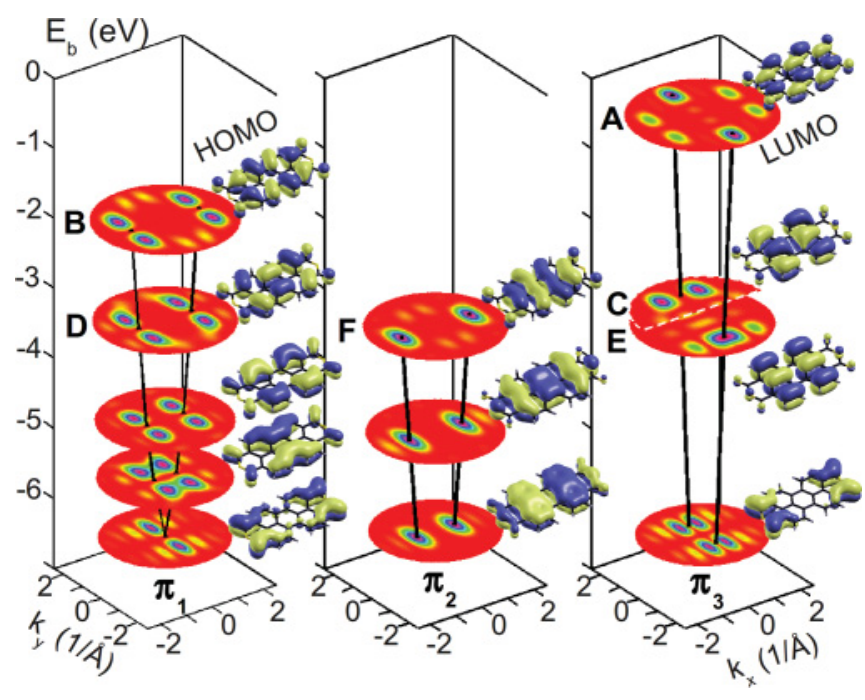

FIG. 3. (Color online) Molecular orbitals of free PTCDA together with the corresponding calculated $\left(k_{x}, k_{y}\right)$ maps of 12 PTCDA $\pi$ orbitals in a binding-energy window between -7 and $0 \mathrm{eV}$. They are grouped into three bands: $\pi_{1}$ (left panel) dispersing along the $k_{x}$ direction and representing orbitals with an $x z$ nodal plane, $\pi_{2}$ (middle panel) dispersing along $k_{x}$ with no node in the $x z$ plane, and $\pi_{3}$ (right panel) dispersing along $k_{y}$ with an increasing number of nodal planes perpendicular to $y$.

increasing energies, similar to what has been observed for other $\pi$-conjugated organic molecules. ${ }^{12,13}$ The common motif of the $\pi_{1}$ momentum maps, however, remains valid across the whole band: four main peaks, symmetrically arranged around $\Gamma$, no intensity in directions $k_{x}=0$ and $k_{y}=0$, and maxima in the $k_{y}$ direction at approximately $0.8 \AA^{-1}$ reflecting the nodal structure of the orbitals perpendicular to the short molecular axis, $y$. The second band, $\pi_{2}$, has no node in the $x z$ plane. Therefore the momentum maps exhibit an intensity maximum along the $k_{y}=0$ line, with pronounced peaks at $k_{x}$ values corresponding to the number of nodal planes perpendicular to the $x$ axis, which are 3, 4, and 5 for the orbitals shown in Fig. 3 . Finally, band $\pi_{3}$ comprises four orbitals with an increasing number of nodal planes in the $x z$ plane; hence the positions of the main peaks in the momentum maps shift toward higher $k_{y}$ values when moving up in energy. The top of this band, the PTCDA LUMO, has four nodal planes in the $y$ direction, resulting in a $k_{y}$ value of about $1.5 \AA^{-1}$.

\section{Momentum space tomography}

We now use these characteristic momentum patterns to search for particular orbitals in our data set. To this end, we plot the experimental photoelectron intensity at constant binding energy (CBE mode) as a function of parallel momenta $k_{x}$ and $k_{y}$ after conversion from polar and azimuthal angles according to Eqs. (1) and (2). Such CBE maps at $E_{b}=-0.8 \mathrm{eV}$ and $E_{b}=-1.9 \mathrm{eV}$ are displayed in Fig. 4, in comparison to the Fourier transform of the LUMO and HOMO of free PTCDA. ${ }^{14}$ Note that we observe an overall broadening in energy of M1 and M2, but specific $E(k)$ dispersion is absent for PTCDA/Ag(110) in contrast to other interfaces of organic monolayers with metallic surfaces. ${ }^{4,46,47}$ Although there are subtle differences between experiment and theory, which must 

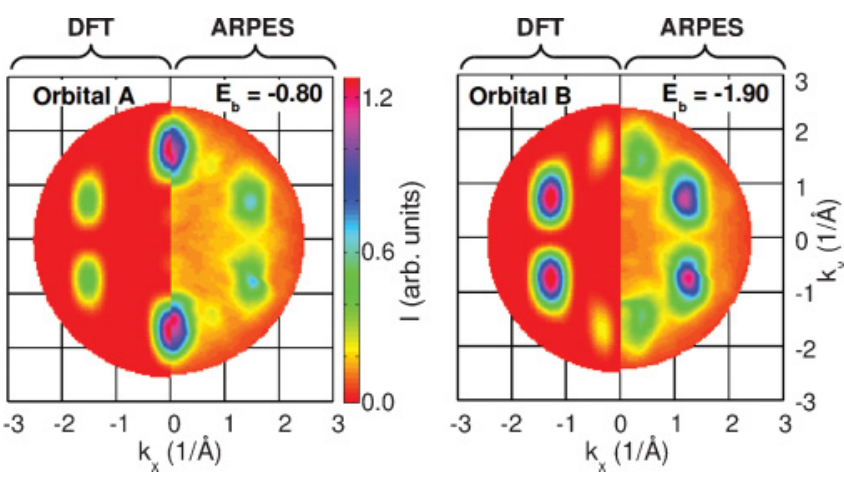

FIG. 4. (Color online) Momentum maps of the molecular features M1 and M2, respectively, identified to be the filled LUMO (left) and HOMO (right) of PTCDA. In each momentum map, theoretical results for a free PTCDA molecule (left halves of maps) are compared to experimental CBE maps (right halves of maps).

reflect differences in the electron distribution between that of the calculated free molecule and the chemisorbed system, their overall agreement proves the assignment of M1 and M2 to the filled LUMO and the HOMO, respectively, which is in accordance with previous studies. $3,15,48$

However, when comparing the earlier momentum maps of the LUMO ${ }^{15}$ with those of Fig. 4 more closely, there are small deviations. In particular, our ARPES data for the LUMO resembles the free-molecule FT more closely than the ARPES data of Ref. 15. Both the feature at normal emission as well as the peak at $0.9 \AA^{-1}$ along $k_{y}$ are much less pronounced in our measurement. One reason could be the different photon energy, $30 \mathrm{eV}$ in our case and $21.2 \mathrm{eV}$ in Ref. 15, leading to the fact that different regions of Fourier space in the $k_{z}$ direction are probed such that the $s$-like charge distribution reported in Ref. 15 is not seen. Another possibility could be the different experimental geometry: Our experiments use photons at a fixed angle of incidence and polarized in the emission plane, while Ziroff et al. utilize unpolarized light for which the angle of incidence is not kept constant. Finally, contributions from uncovered areas of the substrate also cannot be excluded as the origin of the $\Gamma$ emission observed previously (see supporting information ${ }^{49}$ ). We hope to provide a more definite explanation in the future when we investigate these influences systematically by varying the photon energy and angle of incidence.

In this paper, we focus on the molecular feature M3, ${ }^{11}$ whose assignment is more complicated and requires the analysis of tomographic sections through $\left(E_{b}, k_{x}, k_{y}\right)$ space. In the remainder of the paper we will illustrate the power of the tomographic method by clarifying the nature of M3. In Fig. 3 possible candidates for the molecular emission M3 are labeled by letters $\mathrm{C}-\mathrm{F}$, following the energetic order of GGA and $G W$ calculations for a free molecule (A denotes the LUMO, and $\mathrm{B}$ the HOMO). Although orbitals $\mathrm{C}$ to $\mathrm{F}$ are calculated to be so close energetically as to be experimentally unresolvable, they can be distinguished by their distinct patterns in momentum space. This is illustrated in Figs. 5(a)5(d), where we compare calculated momentum maps for these orbitals with experimental CBE maps in the $\left(k_{x}, k_{y}\right)$ plane. In the binding-energy range from -3.0 to $-3.8 \mathrm{eV}$, we have recorded $45 \mathrm{CBE}$ maps at a spacing of $0.02 \mathrm{eV}$, yielding a rather dense $I\left(E_{b}, k_{x}, k_{y}\right)$ data cube ( $I=$ photoelectron intensity).

Three orbitals are found easily by inspection. For instance, orbital $\mathrm{F}$ is the only one among the candidates $\mathrm{C}$ to $\mathrm{F}$ that is expected to show PE intensity along the $k_{y}=0$ line. Moreover, on this line the peak intensity should occur at $k_{x}=1.3 \AA^{-1}$ (Fig. 3). Analyzing tomographic EDC scans $I\left(E_{b}, k_{x}, k_{y}=\right.$ $0)$ and $I\left(E_{b}, k_{x}=1.3 \AA^{-1}, k_{y}\right)$ through our experimental data cube $I\left(E_{b}, k_{x}, k_{y}\right)$ [Fig. 5(a)], we find that the photoemission corresponding to F peaks at $E_{b}=-3.25 \mathrm{eV}$. Indeed, the CBE section through the data cube at this binding energy reveals (among other contributions) the pattern of $\mathrm{F}$, allowing us to identify this orbital in the low binding-energy tail of M3. At $0.13 \mathrm{eV}$ higher in binding energy, the character of the CBE map has changed considerably [Fig. 5(b)]. Here the maximum around $\left(k_{x}, k_{y}\right)=(0.8,1.15) \AA^{-1}$ is the most pronounced one, while traces of orbital $\mathrm{F}$ are still visible. With the help of tomographic EDC scans at $k$ values characteristic for orbital C, i.e., $k_{x}=0.8 \AA^{-1}$ and $k_{y}=1.15 \AA^{-1}$ [Fig. 5(b)], we assign the emission centered around $E_{b}=-3.38 \mathrm{eV}$ to orbital C. When moving a further $0.15 \mathrm{eV}$ to higher binding energy [Fig. 5(c)], we can clearly recognize orbital $\mathrm{E}$ as a third contribution to $\mathrm{M} 3$. It can be distinguished from $\mathrm{F}$ and $\mathrm{C}$ by its intensity along the $k_{x}=0$ line, where both $\mathrm{F}$ and $\mathrm{C}$ have vanishing emission probability. Using tomographic EDC scans along this direction and additionally at $k_{y}=1.15 \AA^{-1}$, we determine the binding energy of $\mathrm{E}$ to be peaked at $-3.53 \mathrm{eV}$. Due to its energetic neighborhood to $\mathrm{C}$, traces of $\mathrm{E}$ can be found in the respective maps and vice versa. Although the overall intensity of emissions from orbital $\mathrm{E}$ seems to be weaker than those from $\mathrm{F}$ and $\mathrm{C}$, it is, nevertheless, clear that the high binding-energy tail of M3 is due to orbital E. In particular, the tomographic EDC scan $I\left(E_{b}, k_{x}, k_{y}=1.15 \AA^{-1}\right)$ depicted in Fig. 5(c) shows the evolution of the two-peak structure at low binding energies that is characteristic for $\mathrm{C}$ to the three-peak structure at larger binding energies that is only found for $\mathrm{E}$.

\section{Deconvolution of photoemission spectra}

Despite its success in identifying three molecular orbitals in M3, the analysis by inspection as described in the previous section has two shortcomings: First, the qualitative nature of the search for characteristic patterns in the $I\left(E_{b}, k_{x}, k_{y}\right)$ data cube means that weaker orbital emissions may be missed, and, second, the analysis only yields the energy positions of the contributing orbitals with no information gained about their energy widths. Fortunately, it is straightforward to generalize the tomographic analysis, such that energy widths can be extracted from the data too. At the same time, this generalization makes the search for contributing orbitals much more systematic, quantitative, and less subjective. We define the sum of least squares of the measured ARPES intensity $I\left(E_{b}, k_{x}, k_{y}\right)$ and a fit function that is a linear combination of the calculated momentum maps $\phi_{i}\left(k_{x}, k_{y}\right)$ of orbital $i$ in the following way:

$$
\chi^{2}=\int d k_{x} d k_{y}\left[I\left(E_{b}, k_{x}, k_{y}\right)-\sum_{i} a_{i}\left(E_{b}\right) \phi_{i}\left(k_{x}, k_{y}\right)\right]^{2} .
$$



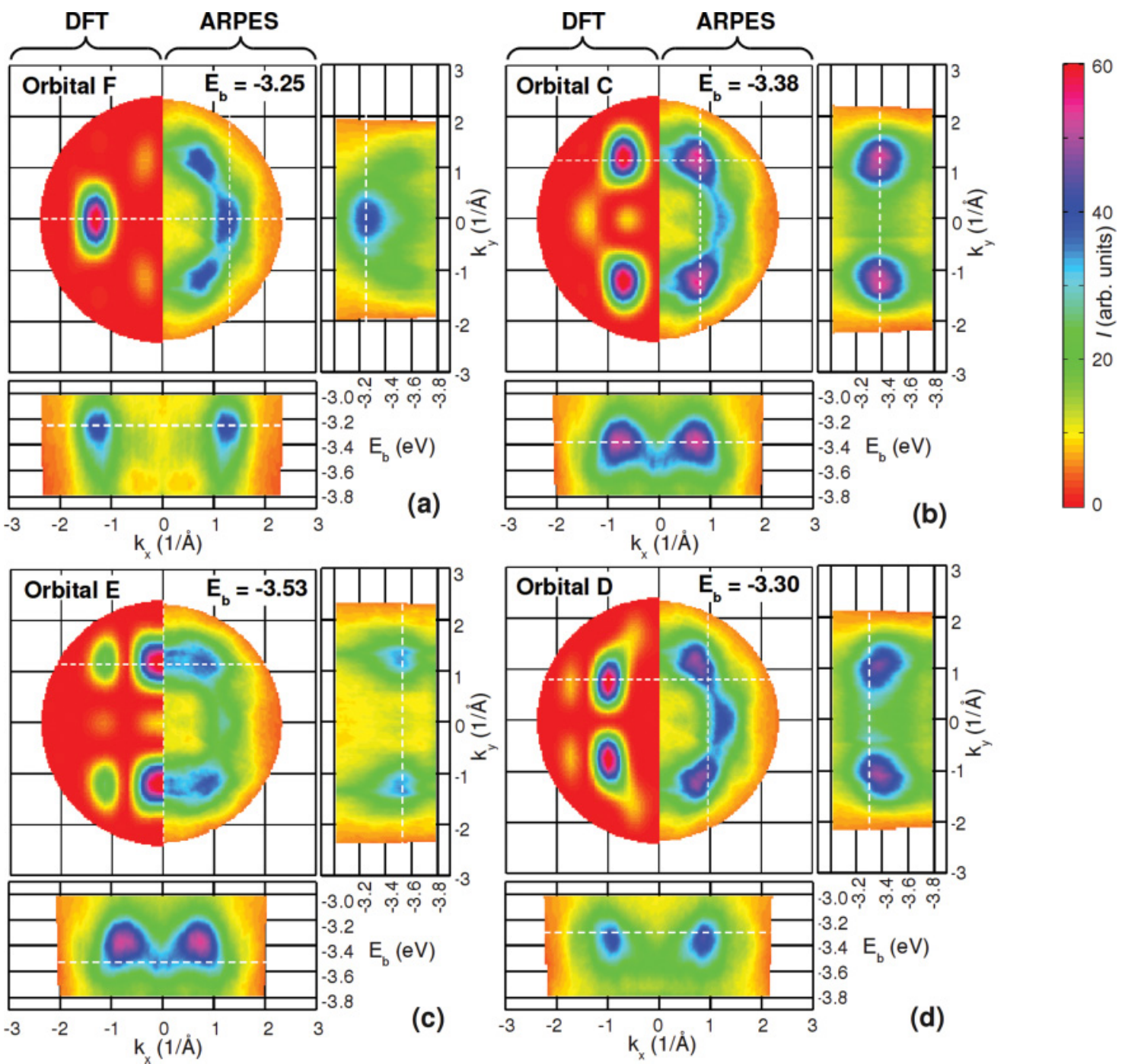

FIG. 5. (Color online) CBE maps and tomographic EDC scans in the binding-energy range between -3.0 and $-3.8 \mathrm{eV}$ corresponding to molecular feature M3. (a) Theoretical momentum map of orbital F (left half) compared to CBE map at $E_{b}=-3.25 \mathrm{eV}$ (right half) and tomographic EDC scans at $k_{y}=0$ (bottom) and $k_{x}=1.3 \AA^{-1}$ (right) as indicated by the white dashed lines. (b) Same as (a) for orbital C at $E_{b}=-3.38 \mathrm{eV}$ with tomographic EDC scans at $k_{y}=1.15$ and $k_{x}=0.8 \AA^{-1}$. (c) Same as (a) for orbital E at $E_{b}=-3.53 \mathrm{eV}$ with tomographic EDC scans at $k_{y}=1.15$ and $k_{x}=0 \AA^{-1}$. (d) Same as (a) for orbital D at $E_{b}=-3.30 \mathrm{eV}$ with tomographic EDC scans at $k_{y}=0.80$ and $k_{x}=0.95 \AA^{-1}$.

By minimizing $\chi^{2}$, we determine the fit coefficients $a_{i}\left(E_{b}\right)$, which can be interpreted as the energy-resolved DOS projected onto molecular orbital $i$. Note that Eq. (4) represents a linear fit problem that results in a linear system of equations for the fit parameters $a_{i}\left(E_{b}\right)$, which can be solved for each energy $E_{b}$ separately. These coefficients are plotted in Fig. 6(a) and compared with the total energy-resolved DOS (shaded area). Note that we have included orbitals $\mathrm{C}-\mathrm{F}$ in the expansion of Eq. (4). The inclusion of orbital D significantly improves the fit compared to only using $\mathrm{C}, \mathrm{E}$, and $\mathrm{F}$, thereby demonstrating that orbital D is also contained in M3. Indeed, by inspecting CBE slices in the tomograph around $E_{b}=-3.30 \mathrm{eV}$ more carefully, orbital D can also be recognized [Fig. 5(d)]. The peak positions obtained for the other three orbitals agree with those determined from the observations in the previous section. In order to check the stability of the fitting procedure, we have also included orbitals A (LUMO) and B (HOMO) in the fit for M3 resulting in no further improvement of the fit and only minor and featureless $a_{A}\left(E_{b}\right)$ and $a_{B}\left(E_{b}\right)$ contributions. As a note, we have also subjected the molecular emissions M1 and M2 to the same fitting procedure. As expected, this has shown that only orbitals A and B contribute to M1 and M2, respectively, while orbitals $\mathrm{C}-\mathrm{F}$, when included in the fit, result in no further improvement of the fit.

In addition to the experimentally deconvoluted DOS, Fig. 6(b) displays, from bottom to top, the calculated orbital energies $\mathrm{C}-\mathrm{F}$ of the free molecule $(G W, \mathrm{SIC}, \mathrm{HSE}$, and GGA), the distorted free molecule (GGA) as well as the molecular-orbital projected DOS for the adsorbed monolayer PTCDA/Ag(110). Compared to experiment, none of the freemolecule calculations is able to predict the correct energetic order among the four $\pi$ orbitals $\mathrm{C}-\mathrm{F}$. In particular, orbital $\mathrm{F}$ exhibits the lowest binding energy in the measurement, while it is at the highest binding energy in the calculations. 


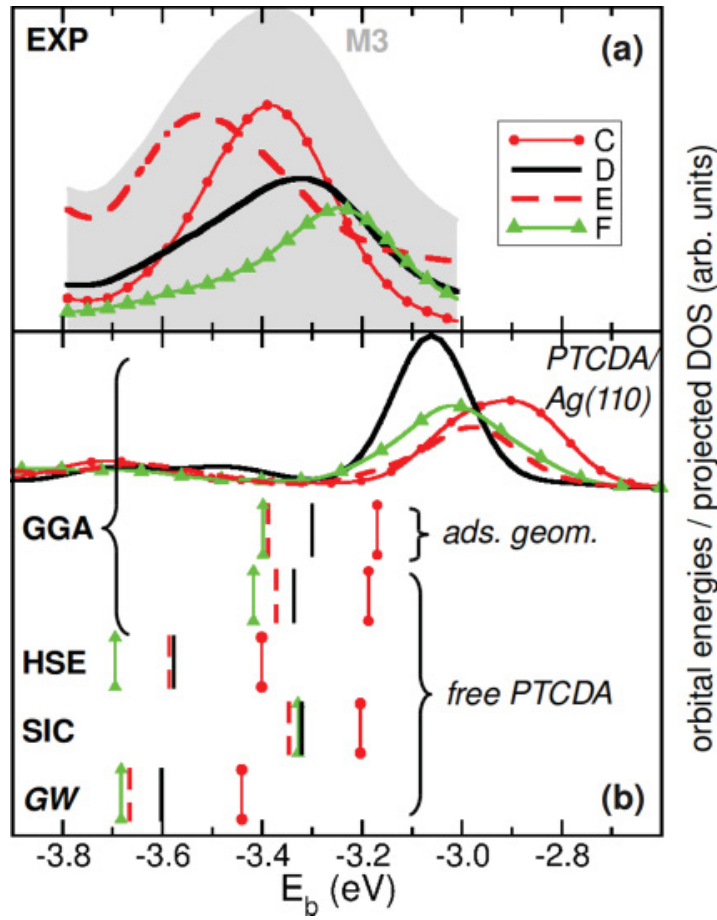

FIG. 6. (Color online) (a) Experimental orbital-projected DOS curves for orbitals C, D, E, and F as obtained least-squares fitting according to Eq. (4). (b) Computed energies of orbitals $\mathrm{C}-\mathrm{F}$ for the free molecule (GGA, HSE, SIC, and $G W$ ), free molecule in adsorption geometry (GGA), and the DOS projected onto $\mathrm{C}-\mathrm{F}$ for the PTCDA/Ag(110) monolayer (GGA).

Similarly, C, the orbital with the lowest computed binding energy, is observed at the second highest binding energy. Since the theoretical orbital ordering also remains unaltered for the free PTCDA calculation using the distorted geometry of the adsorbed molecule, we attribute the measured orbital ordering to metal bonding whose strength depends on the shape and symmetry of the involved molecular orbital. To this end, we analyze the GGA calculation of the adsorbed system

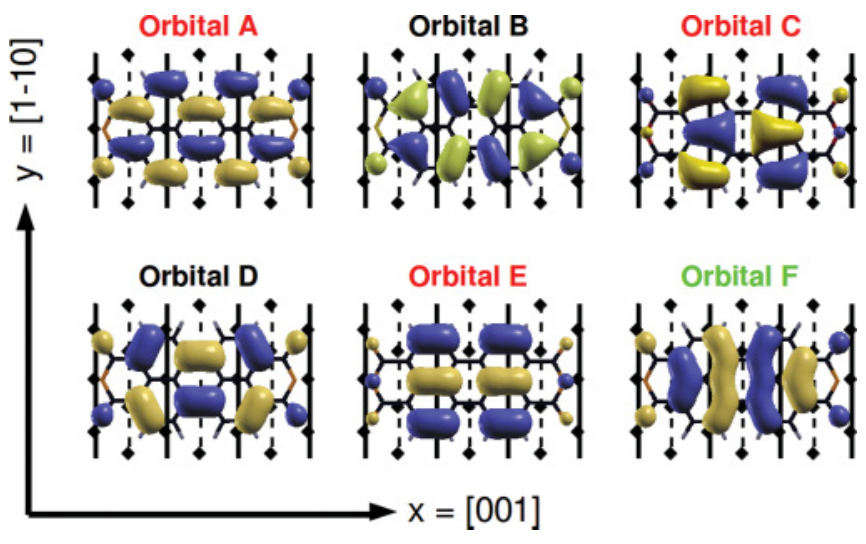

FIG. 7. (Color online) Orbitals A-F of the free PTCDA molecule atop the $\mathrm{Ag}(110)$ substrate. The $\mathrm{Ag}$ rows are running in the [1-10] direction and are spaced by one Ag silver lattice parameter in the [001] direction; the topmost rows are indicated by the thick lines, the second-layer rows by the dashed lines. The position of the silver atoms in these rows are shown as diamond symbols. [lines in Fig. 6(b)]. This orbital-projected DOS shows some modification compared to the free molecule GGA result. Most notably, orbital F no longer has the highest binding energy as in all free-molecule calculations, but is destabilized due to molecule-substrate interactions.

The experimentally deduced ordering can be viewed as a shift of the $\pi_{3}$ band (orbitals $\mathrm{C}$ and $\mathrm{E}$ ) to higher binding energy relative to the orbitals $D$ ( $\pi_{1}$ band), and $F\left(\pi_{2}\right.$ band). Such a bond stabilization of $\mathrm{C}$ and $\mathrm{E}$ with respect to $\mathrm{D}$ and $\mathrm{F}$ might be rationalized by their nodal patterns. $\mathrm{C}$ and $\mathrm{E}$ have lobes positioned on the underlying Ag surface atoms, while the orbitals D and $\mathrm{F}$ exhibit nodes along the topmost $\mathrm{Ag}$ rows as is visualized in Fig. 7. We note, however, that the results at the GGA level are unable to give the ordering observed in the experiment. Its failure may result from its inability to get the relative positions between $\mathrm{Ag} d$ bands and molecular states right. We believe that $G W$ calculations for the adsorbed system could resolve these problems; however, at present such computations are beyond the reach of present computers and codes.

\section{CONCLUSION}

In summary, we have demonstrated a method which allows the deconvolution of valence-band spectra of organic molecular films. Although PTCDA is a two-dimensional molecule and therefore exhibits a more complicated intramolecular band structure than linear molecules, we disentangle its valence band spectrum unambiguously into contributions from six molecular orbitals with a binding energy below $4 \mathrm{eV}$, four of them within an energy range of only $0.4 \mathrm{eV}$, which is in the same order as their intrinsic widths.

This is achieved by employing a tomographic technique that makes use of orbital-characteristic patterns in momentum space. We project the experimental data onto molecular orbitals of the free molecule's orbitals, thus enabling the experimental determination of orbital-projected density-of-state (PDOS) curves, thereby providing a powerful and at the same time simple and easy-to-apply and tool for the analysis of ARPES data. Note that we intentionally use the free molecule's orbitals for this projection as a well-defined reference. Clearly, for the coupled (hybridized) molecule-substrate system, it does not make sense anymore to speak of molecular orbitals, rather all are true eigenstates of the coupled system extended states. By projecting the ARPES momentum maps on free-molecule orbitals, we learn how close the true eigenstates of the coupled system are to the ones of the free molecule and can interpret energy shifts and broadenings in terms of molecule-substrate interactions.

Moreover, by using the full momentum dependence of ARPES, the technique is applicable even if molecular states overlap in energy. It may even allow the extraction of molecular states in regions where the DOS is dominated by strong substrate emission such as metal $d$-bands by using the distinct momentum distributions of molecular and substrate-derived states. We envision a wide applicability of the presented approach for studying the valence-band electronic structure of organic molecular films, particularly with the latest generation of angular-resolved display-type electron spectrometers. This may not only lead to a renaissance of angle-resolved photoemission spectroscopy in the field of organic electronics, 
but it also provides stringent tests for further development of accurate electronic-structure calculations.

\section{ACKNOWLEDGMENTS}

We acknowledge financial support from the Austrian Science Fund (FWF), projects S97-04 and S97-14 and P21330N20 and P23190-N16, and from the Deutsche Forschungsgemeinschaft, project TA-244. We further acknowledge the
Helmholtz-Zentrum-Berlin, Electron storage ring BESSY II for provision of synchrotron radiation at beamline U125/2SGM, and in particular we thank Dr. Christian SchüsslerLangeheine for assistance. We would also like to thank Prof. Falko P. Netzer and Prof. Thomas Seyller for discussions. The research leading to these results has received funding from the European Community's Seventh Framework Programme (FP7/2007-2013) under grant agreement number 226716. *peter.puschnig@unileoben.ac.at

${ }^{1}$ N. Ueno and S. Kera, Prog. Surf. Sci. 83, 490 (2008).

${ }^{2}$ J. Repp, G. Meyer, S. M. Stojkovic, A. Gourdon, and C. Joachim, Phys. Rev. Lett. 94, 026803 (2005).

${ }^{3}$ A. Kraft, R. Temirov, S. K. M. Henze, S. Soubatch, M. Rohlfing, and F. S. Tautz, Phys. Rev. B 74, 041402(R) (2006).

${ }^{4}$ R. Temirov, S. Soubatch, A. Luican, and F. S. Tautz, Nature (London) 444, 350 (2006).

${ }^{5}$ J. Kröger, H. Jensen, R. Berndt, R. Rurali, and N. Lorente, Chem. Phys. Lett. 438, 249 (2007).

${ }^{6}$ I. Torrente, K. Franke, and J. Pascual, J. Phys. Condens. Matter 20, 184001 (2008).

${ }^{7}$ S. Soubatch, C. Weiss, R. Temirov, and F. S. Tautz, Phys. Rev. Lett. 102, 177405 (2009).

${ }^{8}$ N. Dori, M. Menon, L. Kilian, M. Sokolowski, L. Kronik, and E. Umbach, Phys. Rev. B 73, 195208 (2006).

${ }^{9}$ T. Körzdörfer, S. Kümmel, N. Marom, and L. Kronik, Phys. Rev. B 79, 201205(R) (2009).

${ }^{10}$ T. Körzdörfer, S. Kümmel, N. Marom, and L. Kronik, Phys. Rev. B 82, 129903(E) (2010).

${ }^{11}$ M. Dauth, T. Körzdörfer, S. Kümmel, J. Ziroff, M. Wiessner, A. Schöll, F. Reinert, M. Arita, and K. Shimada, Phys. Rev. Lett. 107, 193002 (2011).

${ }^{12}$ G. Koller, S. Berkebile, M. Oehzelt, P. Puschnig, C. AmbroschDraxl, F. P. Netzer, and M. G. Ramsey, Science 317, 351 (2007).

${ }^{13}$ S. Berkebile, P. Puschnig, G. Koller, M. Oehzelt, F. P. Netzer, C. Ambrosch-Draxl, and M. G. Ramsey, Phys. Rev. B 77, 115312 (2008).

${ }^{14}$ P. Puschnig, S. Berkebile, A. J. Fleming, G. Koller, K. Emtsev, T. Seyller, J. D. Riley, C. Ambrosch-Draxl, F. P. Netzer, and M. G. Ramsey, Science 326, 702 (2009).

${ }^{15}$ J. Ziroff, F. Forster, A. Schöll, P. Puschnig, and F. Reinert, Phys. Rev. Lett. 104, 233004 (2010).

${ }^{16}$ M.-H. Shang, M. Nagaosa, S. Nagamatsu, S. Hosoumi, S. Kera, T. Fujikawa, and N. Ueno, J. Electron. Spectr. Rel. Phen. 184, 261 (2011).

${ }^{17}$ L. Broekman, A. Tadich, E. Huwald, J. Riley, R. Leckey, T. Seyller, K. Emtsev, and L. Ley, J. Electron. Spectrosc. Relat. Phenom. 144-147, 1001 (2005).

${ }^{18}$ X. Gonze et al., Comp. Phys. Commun. 180, 2582 (2009).

${ }^{19}$ N. Troullier and J. L. Martins, Phys. Rev. B 43, 1993 (1991).

${ }^{20}$ J. P. Perdew, K. Burke, and M. Ernzerhof, Phys. Rev. Lett. 77, 3865 (1996).

${ }^{21}$ J. Heyd, G. E. Scuseria, and M. Ernzerhof, J. Chem. Phys. 118, 8207 (2003).

${ }^{22}$ J. Heyd, G. E. Scuseria, and M. Ernzerhof, J. Chem. Phys. 124, 219906 (2006).
${ }^{23}$ G. Kresse and J. Hafner, Phys. Rev. B 47, 558 (1993).

${ }^{24}$ G. Kresse and D. Joubert, Phys. Rev. B 59, 1758 (1999).

${ }^{25}$ S. Ismail-Beigi, Phys. Rev. B 73, 233103 (2006).

${ }^{26}$ M. S. Hybertsen and S. G. Louie, Phys. Rev. B 34, 5390 (1986).

${ }^{27}$ J. A. Berger, L. Reining, and F. Sottile, Phys. Rev. B 82, 041103 (2010).

${ }^{28}$ J. W. Gadzuk, Phys. Rev. B 10, 5030 (1974).

${ }^{29}$ E. L. Shirley, L. J. Terminello, A. Santoni, and F. J. Himpsel, Phys. Rev. B 51, 13614 (1995).

${ }^{30}$ A. Mugarza, J. E. Ortega, F. J. Himpsel, and F. J. García de Abajo, Phys. Rev. B 67, 081404 (2003).

${ }^{31}$ F. Himpsel, J. Electron. Spectrosc. Relat. Phenom. 183, 114 (2011).

${ }^{32}$ P. E. Blöchl, Phys. Rev. B 50, 17953 (1994).

${ }^{33}$ H. J. Monkhorst and J. D. Pack, Phys. Rev. B 13, 5188 (1976).

${ }^{34}$ M. Methfessel and A. T. Paxton, Phys. Rev. B 40, 3616 (1989).

${ }^{35}$ J. Neugebauer and M. Scheffler, Phys. Rev. B 46, 16067 (1992).

${ }^{36}$ P. Sony, P. Puschnig, D. Nabok, and C. Ambrosch-Draxl, Phys. Rev. Lett. 99, 176401 (2007).

${ }^{37}$ L. Romaner, D. Nabok, P. Puschnig, E. Zojer, and C. AmbroschDraxl, New J. Phys. 11, 053010 (2009).

${ }^{38}$ O. Bauer, G. Mercurio, M. Willenbockel, S. Soubatch, C. Schmitz, M. Sokolowski, and F. S. Tautz (unpublished).

${ }^{39}$ L. Romaner, G. Heimel, J. L. Bredas, A. Gerlach, F. Schreiber, R. L. Johnson, J. Zegenhagen, S. Duhm, N. Koch, and E. Zojer, Phys. Rev. Lett. 99, 256801 (2007).

${ }^{40}$ G. M. Rangger, L. Romaner, G. Heimel, and E. Zojer, Surf. Interface Anal. 40, 371 (2008).

${ }^{41}$ J. Soler, E. Artacho, J. D. Gale, A. Garcia, J. Junquera, P. Ordejon, and D. Sanchez-Portal, J. Phys. Condens. Matter 14, 2745 (2002).

${ }^{42}$ F. S. Tautz, Prog. Surf. Sci. 82, 479 (2007).

${ }^{43}$ M. Böhringer, W.-D. Schneider, K. Glöckler, E. Umbach, and R. Berndt, Surf. Sci. 419, L95 (1998).

${ }^{44}$ Y. Zou, L. Kilian, A. Schöll, T. Schmidt, R. Fink, and E. Umbach, Surf. Sci. 600, 1260 (2006)

${ }^{45}$ A. Hauschild, K. Karki, B. C. C. Cowie, M. Rohlfing, F. S. Tautz, and M. Sokolowski, Phys. Rev. Lett. 94, 036106 (2005).

${ }^{46}$ H. Yamane, D. Yoshimura, E. Kawabe, R. Sumii, K. Kanai, Y. Ouchi, N. Ueno, and K. Seki, Phys. Rev. B 76, 165436 (2007).

${ }^{47}$ S. Berkebile et al., PhysChemChemPhys 13, 3604 (2011).

${ }^{48}$ K. Glöckler, C. Seidel, A. Soukopp, M. Sokolowski, E. Umbach, M. Bohringer, R. Berndt, and W.-D. Schneider, Surf. Sci. 405, 1 (1998).

${ }^{49}$ See Supplemental Material at http://link.aps.org/supplemental/ 10.1103/PhysRevB.84.235427 for experimental ARPES momentum maps of PTCDA/Ag(110) and of the clean $\operatorname{Ag}(110)$ substrate, respectively. 